\title{
Quantitative evaluation of vision-related and health-related quality of life after endoscopic transsphenoidal surgery for pituitary adenoma
}

\author{
Amparo Wolf, MD, PhD, ${ }^{1}$ Alexandra Coros, BSc, Joel Bierer, BSc, ${ }^{1}$ Sandy Goncalves, MSc, ${ }^{1}$ \\ Paul Cooper, MD, ${ }^{2}$ Stan Van Uum, MD, PhD, ${ }^{3}$ Donald H. Lee, MD, ${ }^{4}$ Alain Proulx, MD, ${ }^{5}$ \\ David Nicolle, MD, ${ }^{2}$ J. Alexander Fraser, MD, ${ }^{2,5}$ Brian W. Rotenberg, MD, MPH, ${ }^{6}$ and \\ Neil Duggal, MD, MSc ${ }^{1}$
}

\begin{abstract}
Divisions of ${ }^{1}$ Neurosurgery and ${ }^{2}$ Neurology, and ${ }^{4}$ Department of Medical Imaging, London Health Sciences Centre; and ${ }^{3}$ Division of Endocrinology and Metabolism, ${ }^{5}$ Department of Ophthalmology, Ivey Eye Institute, and ${ }^{6}$ Department of Otolaryngology-Head \& Neck Surgery, St. Joseph's Hospital, London, Ontario, Canada
\end{abstract}

\begin{abstract}
OBJECTIVE Endoscopic resection of pituitary adenomas has been reported to improve vision function in up to $80 \%-90 \%$ of patients with visual impairment due to these adenomas. It is unclear how these reported rates translate into improvement in visual outcomes and general health as perceived by the patients. The authors evaluated self-assessed health-related quality of life (HR-QOL) and vision-related QOL (VR-QOL) in patients before and after endoscopic resection of pituitary adenomas.
\end{abstract}

METHODS The authors prospectively collected data from 50 patients who underwent endoscopic resection of pituitary adenomas. This cohort included 32 patients (64\%) with visual impairment preoperatively. Twenty-seven patients (54\%) had pituitary dysfunction, including $17(34 \%)$ with hormone-producing tumors. Patients completed the National Eye Institute Visual Functioning Questionnaire and the 36-Item Short Form Health Survey preoperatively and 6 weeks and 6 months after surgery.

RESULTS Patients with preoperative visual impairment reported a significant impact of this condition on VR-QOL preoperatively, including general vision, near activities, and peripheral vision; they also noted vision-specific impacts on mental health, role difficulties, dependency, and driving. After endoscopic resection of adenomas, patients reported improvement across all these categories 6 weeks postoperatively, and this improvement was maintained by 6 months postoperatively. Patients with preoperative pituitary dysfunction, including hormone-producing tumors, perceived their general health and physical function as poorer, with some of these patients reporting improvement in perceived general health after the endoscopic surgery. All patients noted that their ability to work or perform activities of daily living was transiently reduced 6 weeks postoperatively, followed by significant improvement by 6 months after the surgery.

CONCLUSIONS Both VR-QOL and patient's perceptions of their ability to do work and perform other daily activities as a result of their physical health significantly improved by 6 months after endoscopic resection of pituitary adenoma. The use of multidimensional QOL questionnaires provides a precise assessment of perceived outcomes after endoscopic surgery.

https://thejns.org/doi/abs/10.3171/2016.7.JNS16200

KEY WORDS pituitary adenoma; endoscopic transsphenoidal surgery; vision-related quality of life; pituitary surgery

$\mathrm{P}$ ITUITARY tumors account for $10 \%-15 \%$ of primary intracranial tumors. Patients commonly undergo resection of pituitary adenomas for endocrinological reasons or for vision-related symptoms, such as visual field defects, visual acuity changes, or ophthalmoplegia, and may report postoperative improvement in headache sever- ity.$^{19}$ Endoscopic transsphenoidal surgery is routinely used in the management of symptomatic pituitary adenomas ${ }^{15}$ and has been reported to improve vision in up to $80 \%-$ $90 \%$ of patients with visual impairment due to pituitary adenomas. ${ }^{8,14}$ These improvements can manifest within a few days after surgery and continue through the ensuing

ABBREVIATIONS HR-QOL = health-related QOL; NEI-VFQ-25 = National Eye Institute Visual Function Questionnaire-25; QOL = quality of life; SF-36 = 36-Item Short Form Health Survey; VR-QOL = vision-related QOL. SUBMITTED January 23, 2016. ACCEPTED July 8, 2016.

INCLUDE WHEN CITING Published online October 7, 2016; DOI: 10.3171/2016.7.JNS16200. 
weeks, although the exact temporal profile remains unclear. ${ }^{3}$ Similarly, biochemical cure for hormone-producing adenomas has been reported to range between $50 \%$ and $80 \% .6,16$

Few studies have examined how objective improvements in vision and endocrinological status after endoscopic resection translate into patient-perceived outcomes. Assessing the impact of endoscopic pituitary surgery on quality of life (QOL) in a multidimensional fashion, including use of vision-related QOL (VR-QOL) and healthrelated QOL (HR-QOL) questionnaires, may help identify the factors that predict successful outcomes of surgery. To date, the few studies on QOL among patients undergoing endoscopic resection of pituitary adenomas are generally retrospective, do not assess vision-specific QOL, and do not compare preoperative and postoperative outcomes directly., ${ }^{2,7,13,17} \mathrm{~A}$ recent prospective study reported improvements in skull base-specific QOL and sinonasal QOL after endoscopic resection of pituitary adenomas up to 1 year after surgery, although that study did not investigate patient-perceived visual outcomes. ${ }^{11}$

The purpose of the present study was 2-fold: 1) to evaluate the impact of preoperative visual impairment and pituitary dysfunction on HR-QOL and VR-QOL in patients with pituitary adenomas who will undergo pituitary surgery and 2) to evaluate the effect of endoscopic transsphenoidal surgery on HR-QOL and VR-QOL with longitudinal follow-up 6 weeks and 6 months postoperatively.

\section{Methods \\ Study Participants}

For this observational study, reviewed and approved by the research ethics board at London Health Sciences Centre, we prospectively collected data from 50 patients who underwent endoscopic transsphenoidal resection of pituitary adenomas at this institution. All patients provided written informed consent before inclusion in this study. All patients were treated by a multidisciplinary team, consisting of a neurosurgeon, neurologist, otolaryngologist, neuroradiologist, neuroophthalmologist, and endocrinologist. The patients underwent endoscopic transsphenoidal surgery for visual deterioration, endocrinological reasons, or both.

\section{Procedures}

The patients completed neuroophthalmological assessment and visual field analyses with the Humphrey Field Analyzer (Ophthalmic Imaging Systems) before and after surgery. Documentation of visual impairment was based on the neuroophthalmologist's report of visual field defects, reduced visual acuity, or both. None of the patients had progressive visual loss due to ocular or systemic disease. One patient had preexisting visual loss in the left eye from physical trauma in childhood in conjunction with newly progressive visual loss in the right eye.

All patients underwent preoperative contrast-enhanced MRI of the head as well as MRI 6 months postoperatively to assess the extent of resection and of decompression of the optic chiasm. Using the postoperative MRI scans, we defined total resection as no evidence of a tumor, near-total resection as resection of more than $90 \%$ of the tumor, and subtotal resection as resection of less than $90 \%$ of the tumor. Pituitary adenoma size was assessed by determining the longest diameter for all 3 axes. Patients were followed up closely by the endocrinology and neurology teams preoperatively and postoperatively via routine testing of pituitary hormones and their target organ hormones.

Resection was performed jointly with an otolaryngologist and neurosurgeon. Generally, a posterior nasal septectomy was performed to allow binasal access, and the faces of the sphenoid and intersphenoidal septations were drilled away. The dura was incised and the tumor resected. Any complex intraoperative CSF leak was repaired in a layered fashion, consisting of fat and fascia grafts, dura matrix overlay, and a vascularized nasoseptal flap. Eight patients had a large intraoperative or postoperative CSF leak, resulting in placement of a lumbar drain, typically discontinued on Day 5.

\section{Outcome Assessments}

Patients were requested to complete 2 validated questionnaires, the National Eye Institute Visual Function Questionnaire-25 (NEI-VFQ-25) and the 36-Item Short Form Health Survey (SF-36), at 3 time points: preoperatively and 6 weeks and 6 months postoperatively.

\section{NEI-VFQ-25}

The NEI-VFQ-25 consists of 25 questions aimed at measuring VR-QOL based on functional difficulties faced by patients. ${ }^{10}$ Each subscale measures VR-QOL as it relates to visual function and subjective well-being. Each item is assigned to 1 of 12 subscales. These subscales include general health, general vision, ocular pain, near activities, distance activities, social functioning (e.g., seeing how people react to what they say or visiting with people in their homes, parties, or restaurants), mental health (e.g., worry about eyesight, feelings of frustration, reduced control, and worry of embarrassment), role difficulties (e.g., accomplishing less and limited in work and other activities), dependency (e.g., staying home and relying on others), driving, color vision, and peripheral vision. The scores for each subscale range from 0 (worst possible functioning) to 100 (best possible functioning). A composite score is calculated by averaging the scores of all 11 subscales, except the general-health subscale.

\section{SF-36}

The SF-36 questionnaire assesses the general well-being of patients. It consists of 8 subscales each containing 2-10 items: physical function, role-physical, bodily pain, general-health perceptions, vitality, social functioning, role-emotional, and mental health. Scores for each scale range from 0 (maximum disability) to 100 (no disability). Both the mental health and physical function scales of the SF-36 have been shown to be valid measures of mental and physical health, respectively. ${ }^{12,18}$

\section{Statistical Analysis}

Statistical analysis was performed with SPSS 20 soft- 
ware (SPSS, Inc.). Data are expressed as mean \pm SD unless indicated otherwise. Repeated-measures ANOVA and Bonferroni post hoc analyses were performed to determine statistically significant preoperative and 6-week and 6-month postoperative VFQ-25 and SF-36 scores. The Student t-test was used to assess statistical significance of differences between group means. Statistical significance was defined as $\mathrm{p}<0.05$.

\section{Results}

\section{Study Patient Characteristics}

Fifty patients underwent endoscopic transsphenoidal surgery for either progressive visual impairment or hormonal dysfunction. All patients had histopathologically verified pituitary adenomas. The mean age was $56.8 \pm$ 13.5 years, and 28 patients were men and 22 were women. The mean tumor size was $2.45 \pm 1.05 \mathrm{~cm}$ along the longest axis. Five (10\%) of the patients had undergone previous transsphenoidal surgery. Preoperatively, 32 patients $(64 \%)$ had visual acuity or visual field impairment, as determined by the neuroophthalmological assessment. Eighteen patients $(36 \%)$ had partial or complete bitemporal hemianopia, $4(8 \%)$ had quadrantanopia, $3(6 \%)$ had unilateral hemianopia, $3(6 \%)$ had complete unilateral vision loss, $3(6 \%)$ had both visual acuity and field loss, and $1(2 \%)$ had reduced visual acuity with normal visual fields.

Twenty-six (81.2\%) of the 32 patients with visual acuity or visual field impairment had objectively measured improvement in visual function after surgical decompression, as recorded by a neuroophthalmologist and by formal visual field testing; 3 of these patients $(9.4 \%)$ remained permanently impaired in the postoperative period assessed. For 3 patients (9.4\%), no formal follow-up visual field assessment was performed, although these patients reported stable subjective vision. None of the patients had worse vision postoperatively. Postoperative MRI at 6 months indicated total or near-total resection in 41 patients $(82 \%)$ and subtotal resection with residual tissue in 9 patients $(18 \%)$. However, MRI at that time demonstrated good decompression of the optic apparatus in all patients. One of the patients in whom a subtotal resection was achieved underwent radiotherapy (50 Gy in 28 fractions) 1 year postoperatively after documented tumor growth, and the other 8 patients underwent continual monitoring with serial MRI.

Twenty-seven patients (54\%) had preoperative pituitary dysfunction, consisting of hormone-producing tumors or hypopituitarism (hypothyroidism, hypogonadism, or hypocortisolism). Seventeen patients (34\%) had clinically relevant hormone-producing tumors, resulting in acromegaly due to growth hormone-producing tumors in 9 patients (18\%), Cushing's disease due to adrenocorticotropic hormone-producing tumors in 5 patients (10\%), and prolactinoma due to prolactin-producing tumors in 3 patients (6\%). By 6 months postoperatively, $11(40.7 \%)$ of the 27 patients had resolution of their endocrinopathy, and the condition in the remaining 16 patients (59.3\%) remained stable. Fourteen $(82.4 \%)$ of the 17 patients with hormoneproducing tumors had a biochemical cure. Three patients (17.6\%) had persistent acromegaly and received continued Sandostatin treatment postoperatively. Two patients (4\%) had a new-onset pan-hypopituitarism postoperatively that was still present at the 6-month follow-up.

\section{What Is the Impact of Preoperative Visual Impairment on VR-QOL and HR-QOL?}

Using the NEI-VFQ-25 questionnaire, we compared VR-QOL scores among 32 patients (64\%) with and 18 patients $(36 \%)$ without visual impairment preoperatively. Compared with patients without vision defects, patients with visual field defects had statistically significantly worse scores for general vision $(\mathrm{p}<0.001)$, near activities $(\mathrm{p}=0.005)$, mental health $(\mathrm{p}<0.001)$, role difficulties $(p=0.012)$, dependency $(p=0.014)$, driving $(p=0.001)$, and peripheral vision $(\mathrm{p}=0.003)$ (Fig. 1). No such differences were detected for general health, ocular pain, distance activities, social function, or color vision. The mean VR-QOL composite score was $74.3 \pm 22.0$ for the patients with visual impairment and $88.9 \pm 12.4$ for the patients without visual impairment $(\mathrm{p}=0.004)$ (Fig. 1). The mean VR-QOL subscale scores among patients without impairment were similar to previously reported scores in a normal population. ${ }^{13}$ All patients reported low rates on the general-health subscale of the NEI-VFQ-25, irrespective of their visual impairment; the score was $53.1 \pm 24.4$ for vision-impaired patients and $50.0 \pm 35.4$ for patients with no visual impairment.

The HR-QOL scores were evaluated with the SF-36 and are summarized in Table 1. No significant differences were detected for any of the SF-36 subscales between patients with or without preoperative visual impairment.

\section{What Is the Impact of Preoperative Pituitary Hormone Dysfunction on HR-QOL?}

We evaluated HR-QOL of 27 patients (54\%) with and 23 patients $(46 \%)$ without preoperative pituitary hormone dysfunction, including pituitary insufficiency, hormoneproducing tumors, or both. Compared with patients with normal pituitary function, patients with hormone dysfunction preoperatively reported lower scores on the SF36 physical function domain $(66.7 \pm 27.8$ vs $82.3 \pm 20.9$, respectively, $\mathrm{p}=0.027$ ) and general-health perception $(57.1 \pm 26.3$ vs $69.2 \pm 15.3$, respectively, $\mathrm{p}=0.049)$, but not for physical role, bodily pain, vitality, social functioning, emotional role, and mental health (Fig. 2). When we compared physical function and general health of the 17 patients with hormone-producing tumors with those of the 33 other patients, we observed a similar perceived reduction in physical function $(63.5 \pm 28.6$ vs $79.2 \pm 23.0$, respectively, $\mathrm{p}=0.041)$ and general health $(51.9 \pm 29.3$ vs $68.1 \pm 16.1$, respectively, $p=0.014$ ) without any significant changes in the other HR-QOL dimensions.

\section{What Is the Impact of Surgery on VR-QOL in Patients With Preoperative Visual Impairment?}

Table 2 summarizes the mean scores across the different NEI-VFQ-25 subscales preoperatively and at 6 weeks and at 6 months postoperatively for 32 patients with preoperative visual impairment. At both 6 weeks and 6 months postoperatively, these patients had statistically significant improvements in the following subscales: general vision, 


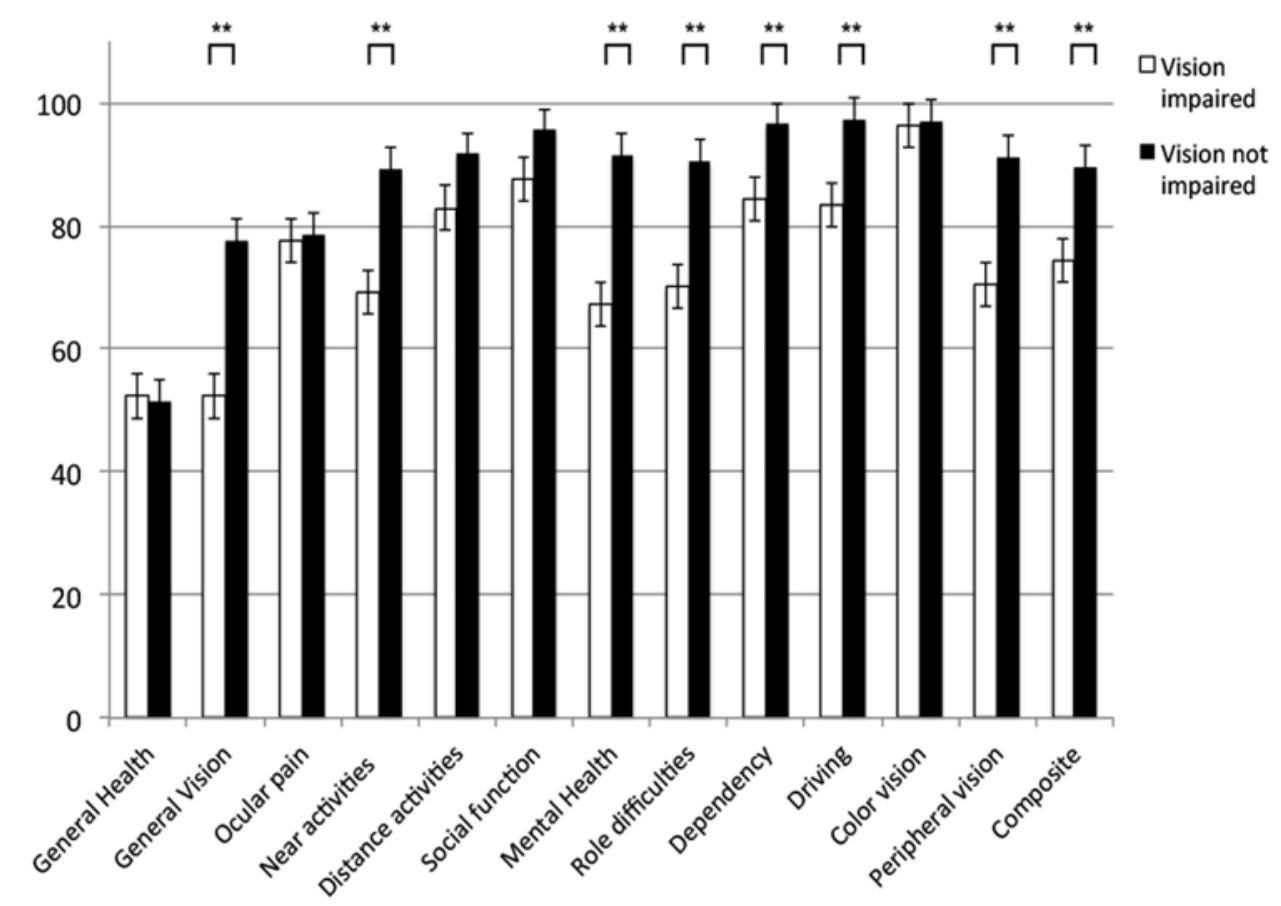

FIG. 1. Mean preoperative NEI-VFQ-25 scores ( \pm standard error) for patients with pituitary adenomas with normal vision and in vision-impaired patients. Brackets with double asterisks indicate statistically significant differences between the 2 patient groups ( $p$ $<0.05)$.

ocular pain, near activities, social functioning, mental health, role difficulties, driving, dependency, peripheral vision, and composite score (Table 2 and Fig. 3). A post hoc Bonferroni test confirmed that all subscale scores statistically significantly changed in the interval between the preoperative period and 6 weeks postoperatively, without any further significant increases between 6 weeks and 6 months postoperatively. General health, distance activities, and color vision did not significantly improve postoperatively. No significant differences were detected in the NEI-VFQ-25 subscale scores at 6 months postoperatively between 27 patients with total or near-total resection and 5 with subtotal resection.

\section{What Is the Impact of Surgery on HR-QOL?}

We assessed the impact of endoscopic transsphenoidal surgery on HR-QOL with the SF-36 (Table 3). A significant change in physical role was detected in the postoperative period $(\mathrm{p}<0.001)$. At 6 weeks postoperatively, physical role, that is, the patients' physical ability to do personal and work-related activities, had significantly declined (preoperatively $61.0 \pm 45.2$ and 6 weeks postoperatively 30.0 $\pm 41.0, \mathrm{p}=0.002$, post hoc Bonferroni test). By 6 months postoperatively, patients reported an improvement in physical role, indicated by scores significantly higher than those measured preoperatively $(6$ months postoperatively $77.9 \pm$ 33.9, $\mathrm{p}=0.03$, post hoc Bonferroni test of the preoperative score vs 6 months postoperatively). We observed a similar trend when we included only patients with preoperative visual impairment in the analysis, with a reduction in physical role 6 weeks postoperatively, followed by significant recovery from preoperative scores (preoperatively $57.0 \pm$
45.4, 6 weeks postoperatively $30.4 \pm 41.0$, and 6 months postoperatively $83.4 \pm 27.0, \mathrm{p}=0.05$, for preoperatively vs 6 weeks postoperatively and $p=0.007$ for preoperatively vs 6 months postoperatively in post hoc Bonferroni tests).

In addition, at 6 weeks postoperatively, we observed a significant decrease in both physical function and social function, followed by recovery back to preoperative scores by 6 months postoperatively (Table 3 ). Patients reported that their general health had improved by 6 weeks postoperatively, but general health returned to preoperative levels only by 6 months postoperatively. No other significant changes were observed in any of the other SF-36 dimensions.

As noted above, patients with pituitary dysfunction, including involvement of hormone-producing tumors, had

TABLE 1. Summary of SF-36 scores activities for patients with and without preoperative impairments

\begin{tabular}{lcc}
\hline \multirow{2}{*}{$\begin{array}{c}\text { SF-36 } \\
\text { Score }\end{array}$} & \multicolumn{2}{c}{ Patient Group } \\
\cline { 2 - 3 } & Visual Impairment & No Visual Impairment \\
\hline Bodily pain & $68.6(22.8)$ & $65.7(33.1)$ \\
\hline Mental health & $75.9(17.9)$ & $76.0(19.6)$ \\
\hline Physical function & $77.0(23.3)$ & $68.3(29.8)$ \\
\hline Role-emotional & $76.0(36.2)$ & $81.5(34.7)$ \\
\hline Role-physical & $57.0(45.4)$ & $68.1(45.2)$ \\
\hline Social functioning & $81.8(18.5)$ & $76.5(28.4)$ \\
\hline General health & $65.8(15.9)$ & $57.1(31.0)$ \\
\hline Vitality & $48.9(22.0)$ & $56.4(24.8)$ \\
\hline
\end{tabular}

Data are presented as the mean $( \pm S D)$. 


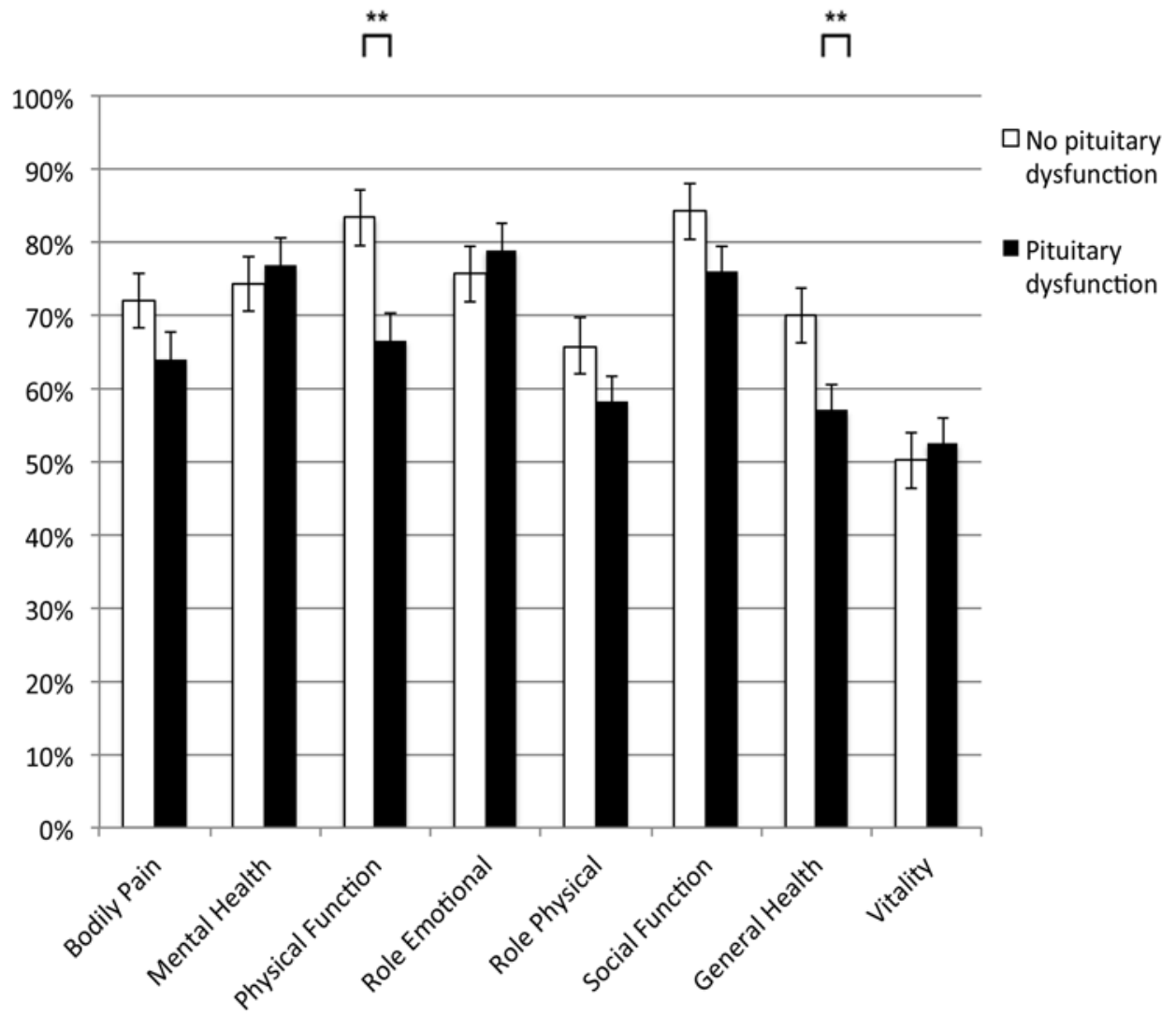

FIG. 2. Mean preoperative SF-36 scores ( \pm standard error) for patients with pituitary adenoma and pituitary dysfunction and for patients with normal pituitary function. Brackets with double asterisks indicate statistically significant differences between the 2 patient groups $(p<0.05)$.

lower perceived physical function and general health than did patients with normal pituitary function preoperatively. No statistically significant improvement in physical function was observed after surgery in 27 patients with pitu- itary dysfunction (the physical function score was $66.7 \pm$ 27.8 preoperatively, $64.1 \pm 26.3$ at 6 weeks postoperatively, and $71.1 \pm 28.0$ at 6 months postoperatively, $\mathrm{F}=1.91, \mathrm{p}=$ 0.31 ). The patients did report mild improvements in their

TABLE 2. Results of repeated-measures ANOVA comparing preoperative and postoperative NEI-VFQ-25 scores for patients with preoperative visual impairment

\begin{tabular}{|c|c|c|c|c|c|}
\hline \multirow[b]{2}{*}{ Variable } & \multicolumn{3}{|c|}{ Time Point } & \multirow{2}{*}{$\begin{array}{c}\mathrm{F} \\
\text { Test }\end{array}$} & \multirow{2}{*}{$\begin{array}{c}p \\
\text { Value }\end{array}$} \\
\hline & Preop & 6 Wks Postop & 6 Mos Postop & & \\
\hline General health & $53.1(24.3)$ & $57.8(17.3)$ & $64.1(23.7)$ & 3.03 & 0.060 \\
\hline General vision & $52.0(27.1)$ & $79.4(14.8)$ & $80.0(16.1)$ & 25.69 & $<0.001$ \\
\hline Ocular pain & $78.1(27.7)$ & $89.8(12.9)$ & $92.0(14.4)$ & 4.63 & 0.030 \\
\hline Near activities & $69.1(25.2)$ & $89.5(17.2)$ & $90.1(16.7)$ & 16.34 & $<0.001$ \\
\hline Distance activities & $82.9(46.3)$ & $92.4(11.3)$ & $93.3(11.9)$ & 1.39 & 0.250 \\
\hline Social functioning & $87.1(20.4)$ & $96.9(11.9)$ & $97.6(9.4)$ & 6.28 & 0.010 \\
\hline Mental health & $67.2(30.1)$ & $89.7(12.5)$ & $92.6(11.9)$ & 17.82 & $<0.001$ \\
\hline Role difficulties & $69.1(32.1)$ & $91.0(18.3)$ & $91.0(21.4)$ & 10.20 & 0.001 \\
\hline Dependency & $83.9(26.1)$ & $96.4(10.8)$ & $97.0(11.1)$ & 6.50 & 0.010 \\
\hline Driving & $83.3(17.2)$ & $94.2(9.1)$ & $91.1(12.2)$ & 4.62 & 0.030 \\
\hline Color vision & $96.1(9.2)$ & $97.7(9.8)$ & $97.1(8.8)$ & 0.49 & 0.610 \\
\hline Peripheral vision & $69.5(28.2)$ & $91.4(15.0)$ & 89.1 (17.9) & 16.41 & $<0.001$ \\
\hline Composite score & $74.4(22.0)$ & $89.8(12.5)$ & $90.4(13.5)$ & 13.88 & $<0.001$ \\
\hline
\end{tabular}

Data represent means $( \pm S D)$ for 32 patients; a $p<0.05$ was considered statistically significant. 


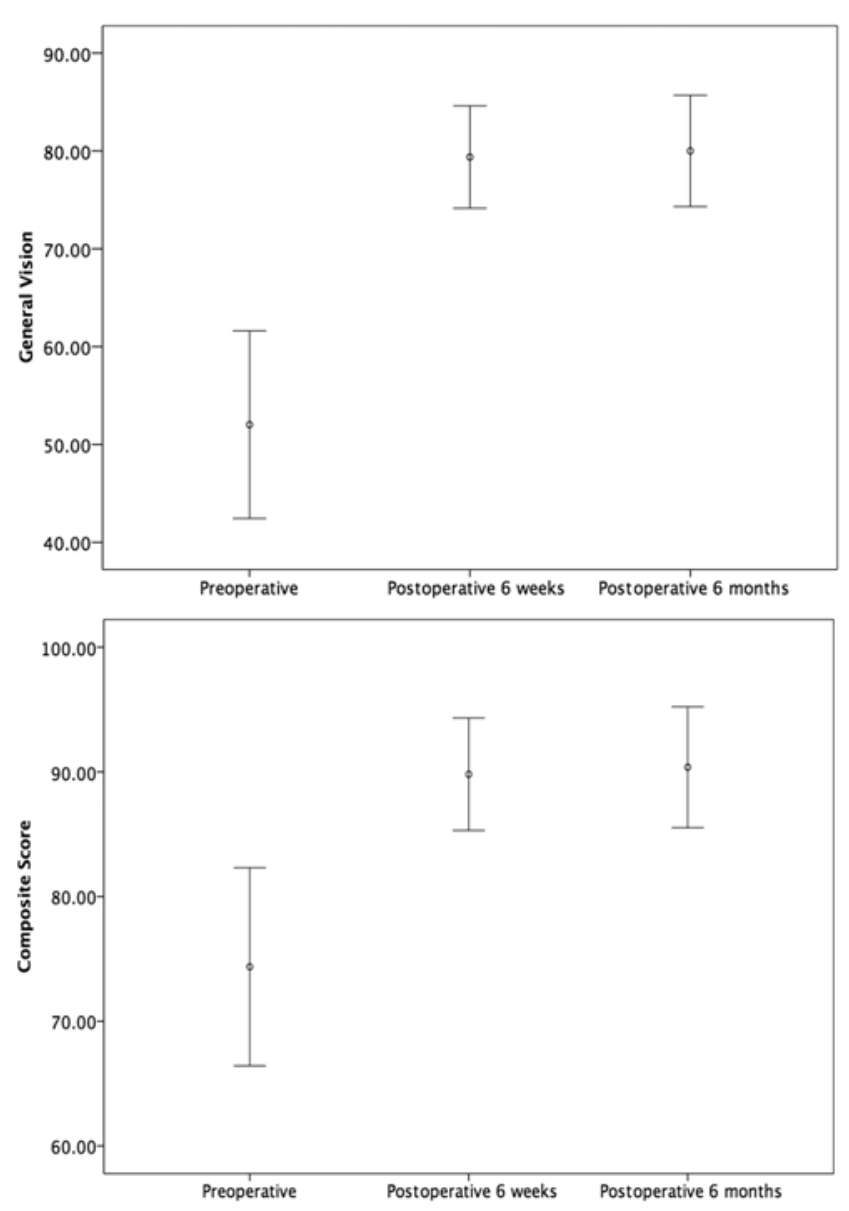

FIG. 3. General vision (upper) and composite (lower) scores determined with the NEI-VFQ-25 preoperatively and at 6 weeks and 6 months postoperatively in patients with visual impairment preoperatively (data are mean $\pm 95 \% \mathrm{Cl}$ ).

general health by 6 weeks postoperatively, and this improvement persisted at 6 months (the general-health score was $57.1 \pm 26.3$ preoperatively, $68.1 \pm 22.3$ at 6 weeks postoperatively, and $64.9 \pm 23.9$ at 6 months postoperatively, $\mathrm{F}$ $=2.98, \mathrm{p}=0.015$; a post hoc Bonferroni test of preoperatively vs 6 weeks postoperatively indicated a $\mathrm{p}=0.012$ ). Surgery did not result in significant changes in bodily pain, vitality, emotional role, and mental health.

\section{Discussion}

The principal finding of this prospective study was that endoscopic pituitary surgery significantly improves perceived visual QOL in patients with preoperative visual impairment by 6 weeks postoperatively and that this improvement is maintained at the 6-month follow-up. Furthermore, by 6 months postoperatively, endoscopic pituitary surgery had improved physical health such that all patients noted an improvement in their ability to work or perform activities of daily living.

Worsening visual function strongly affects driving, role functioning, and mental health and also increases dependence on others. Using the vision-specific NEI-VFQ-25, we observed that compared with patients without preop- erative visual impairment, patients with such impairment had significantly lower NEI-VFQ-25 scores across multiple dimensions, including general vision, near activities, mental health, role difficulties, dependency, peripheral vision, and driving. Previous studies have also reported reduced vision-related QOL in patients with visual impairment due to a variety of disorders, including pituitary adenomas, glaucoma, postischemic injuries, and other causes. ${ }^{4,7,9}$

Few studies have directly investigated the impact of surgery on perceived visual outcomes in patients with pituitary adenomas. Our results indicate that endoscopic transsphenoidal resection of pituitary adenomas in patients with preoperative visual impairment significantly improves perceived general vision, near activities, peripheral vision, and vision-related mental health and role difficulties. Postoperative improvements were more modest for ocular pain, social functioning, and driving. We had expected that patients would have postoperative improvements in general and peripheral vision as well as near activities. Interestingly, patients also reported improvements in their visionspecific mental health, which includes worrying about their eyesight and feelings of frustration and loss of control because of their poor eyesight. Moreover, preoperatively, the patients reported that they felt they accomplished less and were limited in their ability to do work and activities because of impaired vision, conditions that significantly improved as early as 6 weeks after surgery.

Interestingly, patients reported some improvements in ocular pain (defined by the NEI-VFQ as discomfort in and around the eyes) after the surgery; this improvement might have been a reflection of diminished headache-related pain. For example, previous studies have shown improvements in headaches after endoscopic resection of pituitary adenomas. ${ }^{5,19}$ Also noteworthy is that patients did not report a significant impact of their pituitary adenoma on color vision and distance activities, and surgery imparted therefore no significant benefits to these faculties.

A previous study has investigated the impact of surgery on VR-QOL and has shown that surgical intervention yielded improvements in all NEI-VFQ-25 subscales, except for general health and color vision, at 3 months after endoscopic resection of pituitary adenomas..$^{13}$ The authors reported that the most important predictors of postoperative QOL are the extent of visual field disturbance in the better-seeing eye and the composite score on the NEIVFQ-25. $\cdot^{13}$ Although studies have reported objective recovery of visual fields over several years and that most of this recovery occurs in the first 3-6 months after surgery, ${ }^{3}$ we have shown here that improvement in visual QOL occurs as early as 6 weeks after surgery and is maintained at 6 months. No significant differences in VR-QOL among patients with varying extents of resection were observed at 6 months postoperatively; this lack of a difference was likely due to the fact that all patients had evidence on MRI scans of good decompression of the optic apparatus at 6 months postoperatively.

In both the preoperative and postoperative periods, and even after successful surgical treatment, patients with pituitary adenomas have lower HR-QOL than individuals in the normal population., ${ }^{1,7}$ The HR-QOL is affected by various factors, and surgical intervention alone may not 
TABLE 3. Results of repeated-measures ANOVA comparing preoperative and postoperative SF-36 scores

\begin{tabular}{lccccc}
\hline \multirow{2}{*}{$\begin{array}{c}\text { SF-36 } \\
\text { Score }\end{array}$} & Preop & 6 Wks Postop & 6 Mos Postop & $\begin{array}{c}\text { F } \\
\text { Test }\end{array}$ & $\begin{array}{c}p \\
\text { Value }\end{array}$ \\
\cline { 2 - 5 } & $67.6(26.7)$ & $64.2(28.8)$ & $75.1(23.7)$ & 2.77 & 0.07 \\
\hline Bodily pain & $76.0(18.3)$ & $78.7(16.2)$ & $79.0(15.0)$ & 0.64 & 0.52 \\
\hline Mental health & $73.9(25.8)$ & $66.3(23.2)$ & $76.0(25.9)$ & 4.26 & 0.02 \\
\hline Physical function & $78.0(35.4)$ & $74.7(40.7)$ & $80.7(33.0)$ & 0.50 & 0.61 \\
\hline Role-emotional & $61.0(45.2)$ & $30.0(41.0)$ & $77.9(33.9)$ & 23.1 & $<0.001$ \\
\hline Role-physical & $79.9(22.4)$ & $73.0(26.9)$ & $83.8(21.0)$ & 4.80 & 0.01 \\
\hline Social function & $62.7(22.5)$ & $72.5(20.0)$ & $67.2(22.2)$ & 8.50 & $<0.001$ \\
\hline General health & $51.6(23.0)$ & $50.9(22.2)$ & $58.4(23.9)$ & 2.60 & 0.08 \\
\hline Vitality & & & &
\end{tabular}

Data represent means $( \pm S D)$ for 50 patients; a $p<0.05$ was considered statistically significant.

improve it significantly. In the current study, patients with preoperative visual impairment did not significantly differ in HR-QOL from those without visual impairment. Of note, the presence of multiple pituitary deficiencies is a predominant predictor of decreased postoperative HRQOL. ${ }^{2}$ Patients in our study who had preoperative pituitary dysfunction, including hormone-producing tumors, reported reduced physical function, that is, the ability to perform all physical activities, including those of daily living, and lower general-health scores than did patients without pituitary dysfunction. By 6 weeks after surgery, the patients with pituitary dysfunction reported that their general health had improved but not their physical function. This analysis took into account all patients who had preoperative pituitary dysfunction, but not the extent of postoperative endocrinological improvement, which was likely quite variable among the patients. All patients having undergone endoscopic resection reported transiently decreased abilities to perform work-related activities (i.e., physical roles) 6 weeks postoperatively. However, by 6 months postoperatively, patients reported that their ability to perform workrelated activities had improved and was superior to that before surgery.

Overall, the findings of our study demonstrate that surgery promotes differential improvement across subscales assessing VR-QOL and HR-QOL. These findings reinforce the importance of multidimensional QOL assessment tools for better predicting the time needed to recover postoperatively and for educating patients on these expected recoveries. Vision-related changes need to be assessed by vision-specific QOL questionnaires rather than by general-health QOL questionnaires. According to our results, patients can expect to experience improvement in visionspecific activities as well as vision-specific mental health by 6 weeks after surgery. Patients with endocrinological dysfunction had reduced general-health QOL, which may improve to some extent by 6 months after surgery.

\section{Study Limitations}

The purpose of the present study was to examine patient-reported outcomes rather than objective visual outcomes. Specifically, this study did not assess any relationships between objectively measured visual outcomes with patient-perceived outcomes, although this relationship is the subject of an ongoing study. Furthermore, although this study examined the impact of hormone dysfunction on QOL, the study was not powered sufficiently to investigate more thoroughly the relationship between biochemical cure rates and QOL in functioning adenomas. Hormonal replacement is generally optimal at 6 months or later rather than at 6 weeks after pituitary surgery, and this delay may affect QOL. Similar to analyzing QOL as it relates to vision defects, assessing QOL in patients with endocrinological changes may be more accurate with the use of questionnaires validated specifically for hormonal deficiencies. The long-term impact of surgery on vision-specific and global QOL beyond 6 months also remains unknown.

\section{Conclusions}

Vision-specific QOL among patients with pituitary adenoma significantly improved after transsphenoidal surgery by 6 weeks postoperatively, and these improvements persisted by 6 months postoperatively. Furthermore, the patients' perceived ability to perform work-related tasks also improved by 6 months postoperatively. Identifying factors that affect multidimensional QOL is important for planning treatment, setting realistic patient expectations, and predicting outcomes, including the effect of pituitary tumors on vision, the progression of visual deficits, and responses of patients' conditions to therapy.

\section{References}

1. Biermasz NR, van Thiel SW, Pereira AM, Hoftijzer HC, van Hemert AM, Smit JWA, et al: Decreased quality of life in patients with acromegaly despite long-term cure of growth hormone excess. J Clin Endocrinol Metab 89:5369-5376, 2004

2. Dekkers OM, van der Klaauw AA, Pereira AM, Biermasz NR, Honkoop PJ, Roelfsema F, et al: Quality of life is decreased after treatment for nonfunctioning pituitary macroadenoma. J Clin Endocrinol Metab 91:3364-3369, 2006

3. Fraser CL, Biousse V, Newman NJ: Visual outcomes after treatment of pituitary adenomas. Neurosurg Clin N Am 23:607-619, 2012

4. Gall C, Franke GH, Sabel BA: Vision-related quality of life in first stroke patients with homonymous visual field defects. Health Qual Life Outcomes 8:33, 2010

5. Hayashi Y, Kita D, Iwato M, Fukui I, Oishi M, Tsutsui T, et al: Significant improvement of intractable headache after transsphenoidal surgery in patients with pituitary adenomas; 
preoperative neuroradiological evaluation and intraoperative intrasellar pressure measurement. Pituitary 19:175-182, 2016

6. Hazer DB, Işı1k S, Berker D, Güler S, Gürlek A, Yücel T, et al: Treatment of acromegaly by endoscopic transsphenoidal surgery: surgical experience in 214 cases and cure rates according to current consensus criteria. J Neurosurg 119:1467-1477, 2013

7. Johnson MD, Woodburn CJ, Vance ML: Quality of life in patients with a pituitary adenoma. Pituitary 6:81-87, 2003

8. Kayan A, Earl CJ: Compressive lesions of the optic nerves and chiasm. Pattern of recovery of vision following surgical treatment. Brain 98:13-28, 1975

9. Kerkhoff G: Restorative and compensatory therapy approaches in cerebral blindness-a review. Restor Neurol Neurosci 15:255-271, 1999

10. Mangione CM, Lee PP, Gutierrez PR, Spritzer K, Berry S, Hays RD: Development of the 25-Item National Eye Institute Visual Function Questionnaire. Arch Ophthalmol 119:1050-1058, 2001

11. McCoul ED, Bedrosian JC, Akselrod O, Anand VK, Schwartz TH: Preservation of multidimensional quality of life after endoscopic pituitary adenoma resection. J Neurosurg 123:813-820, 2015

12. McHorney CA, Ware JE Jr, Raczek AE: The MOS 36-Item Short-Form Health Survey (SF-36): II. Psychometric and clinical tests of validity in measuring physical and mental health constructs. Med Care 31:247-263, 1993

13. Okamoto Y, Okamoto F, Yamada S, Honda M, Hiraoka T, Oshika T: Vision-related quality of life after transsphenoidal surgery for pituitary adenoma. Invest Ophthalmol Vis Sci 51:3405-3410, 2010

14. Peter M, De Tribolet N: Visual outcome after transsphenoidal surgery for pituitary adenomas. Br J Neurosurg 9:151-157, 1995

15. Rotenberg B, Tam S, Ryu WHA, Duggal N: Microscopic versus endoscopic pituitary surgery: a systematic review. Laryngoscope 120:1292-1297, 2010

16. Shin SS, Gardner PA, Ng J, Faraji AH, Agarwal N, Chivukula S, et al: Endoscopic endonasal approach for ACTH-secreting pituitary adenomas: outcomes and analysis of remission rates and tumor biochemical activity with respect to tumor invasiveness. World Neurosurg [epub ahead of print], 2015

17. Tanemura E, Nagatani T, Aimi Y, Kishida Y, Takeuchi K, Wakabayashi T: Quality of life in nonfunctioning pituitary macroadenoma patients before and after surgical treatment. Acta Neurochir (Wien) 154:1895-1902, 2012

18. Ware JE Jr, Gandek B: Overview of the SF-36 Health Survey and the International Quality of Life Assessment (IQOLA) Project. J Clin Epidemiol 51:903-912, 1998

19. Wolf A, Goncalves S, Salehi F, Bird J, Cooper P, Van Uum S, et al: Quantitative evaluation of headache severity before and after endoscopic transsphenoidal surgery for pituitary adenoma. J Neurosurg 124:1627-1633, 2016

\section{Disclosures}

The authors report no conflict of interest concerning the materials or methods used in this study or the findings specified in this paper.

\section{Author Contributions}

Conception and design: Duggal, Cooper, van Uum, Rotenberg. Acquisition of data: Duggal, Wolf, Coros, Goncalves, Cooper, van Uum, Lee, Proulx, Nicolle, Fraser, Rotenberg. Analysis and interpretation of data: Duggal, Wolf, Bierer, Goncalves, Cooper, van Uum, Lee, Proulx, Nicolle, Fraser, Rotenberg. Drafting the article: Duggal, Wolf, Bierer, Goncalves, van Uum, Rotenberg. Critically revising the article: Duggal, Wolf, Bierer, Goncalves, Cooper, van Uum, Lee, Proulx, Nicolle, Fraser, Rotenberg. Reviewed submitted version of manuscript: all authors. Approved the final version of the manuscript on behalf of all authors: Duggal. Statistical analysis: Wolf, Goncalves. Study supervision: Duggal.

\section{Correspondence}

Neil Duggal, London Health Sciences Centre, University Hospital, 339 Windermere Rd., London, ON N6A 5A5, Canada. email: neil.duggal@1hsc.on.ca. 